\title{
Review of cavity optomechanical cooling *
}

\author{
Yong-Chun Liu ${ }^{\mathrm{a}) \dagger}, \quad \mathrm{Yu}-\mathrm{Wen} \mathrm{Hu}^{\mathrm{a})}$ ， Chee Wei Wong ${ }^{\mathrm{b})}$ and Yun-Feng Xiao ${ }^{\mathrm{a}) \ddagger}$ \\ a) State Key Laboratory for Mesoscopic Physics and School of Physics, Peking University, Beijing 100871, \\ China \\ b) Optical Nanostructures Laboratory, Columbia University, New York, New York 10027, USA
}

\begin{abstract}
Quantum manipulation of macroscopic mechanical systems is of great interest in both fundamental physics and applications ranging from high-precision metrology to quantum information processing. A crucial goal is to cool the mechanical system to its quantum ground state. In this review, we focus on the cavity optomechanical cooling, which exploits the cavity enhanced interaction between optical field and mechanical motion to reduce the thermal noise. Recent remarkable theoretical and experimental efforts in this field have taken a major step forward in preparing the motional quantum ground state of mesoscopic mechanical systems. This review first describes the quantum theory of cavity optomechanical cooling, including quantum noise approach and covariance approach; then the up-to-date experimental progresses are introduced. Finally, new cooling approaches are discussed along the directions of cooling in the strong coupling regime and cooling beyond the resolved sideband limit.
\end{abstract}

Keywords: cavity optomechanics, optomechanical cooling, cavity cooling, ground state cooling, mechanical resonator

PACS: 42.50.Wk, 07.10.Cm, 42.50.Lc

\section{Contents}

1. Introduction

2. Quantum theory of cavity optomechanical cooling

2.1. System Hamiltonian and linearization . . . . . . . . . . . . . . . . 6

2.2. Quantum noise approach . . . . . . . . . . . . . . . . . . . . . . . . . 9

2.3. Covariance approach . . . . . . . . . . . . . . . . . . . . . . 12

${ }^{*}$ We thank Yi-Wen Hu and Xingsheng Luan for helpful discussions. Project supported by 973 program (Grant No. 2013CB328704), National Natural Science Foundation of China (Grant Nos. 11004003, 11222440, and 11121091), and RFDPH (No. 20120001110068). Y.-C. Liu acknowledges the support from the Scholarship Award for Excellent Doctoral Student granted by Ministry of Education.

†E-mail:ycliu@pku.edu.cn

${ }^{\ddagger}$ URL: www.phy.pku.edu.cn/ yfxiao/index.html 


\section{New cooling approaches}

4.1. Cooling in the strong coupling regime . . . . . . . . . . . . . . . . 18

4.2. Cooling beyond the resolved sideband limit . . . . . . . . . . . . . . 20

4.2.1. Novel coupling mechanisms . . . . . . . . . . . . . . . . . 20 20

4.2.2. Parameter modulations . . . . . . . . . . . . . . . . 222

4.2.3. Hybrid systems . . . . . . . . . . . . . . . . . . . . . . 2 22

5. Summary and outlook

\section{Introduction}

Optomechanics is an emerging field exploring the interaction between light and mechanical motion. Such interaction originates from the mechanical effect of light, i.e., optical force. Radiation pressure force (or scattering force) and optical gradient force (or dipole force) are two typical categories of optical forces. The radiation pressure force originates from the fact that light carries momentum. The momentum transfer from light to a mechanical object exerts a pressure force on the object. This was noticed dating back to the 17th century by Kepler, who noted that the dust tails of comets point away from the sun. In the 1970s, Hänsch and Schawlow [1, Wineland and Dehmelt [2] pointed out the possibility of cooling atoms by using radiation pressure force of a laser. This was subsequently realized experimentally [3], and it has now become an important technique for manipulating atoms. The gradient force stems from the electromagnetic field gradient. The nonuniform field polarizes the mechanical object in a way that the positively and negatively charged sides of the dipole experience different forces, leading to nonzero net optical force acting on the object. It was first demonstrated by Ashkin that focused laser beams can be used to trap micro- and nano-scale particles [4. This has stimulated the technique of optical tweezers, which are widely used to manipulate living cells, DNA and bacteria. There are other kinds of optical forces, for instance, photothermal force (bolometric force) [5], which results from the thermalelastic effect.

The optical forces exerting on macroscopic/mesoscopic mechanical objects are typically 


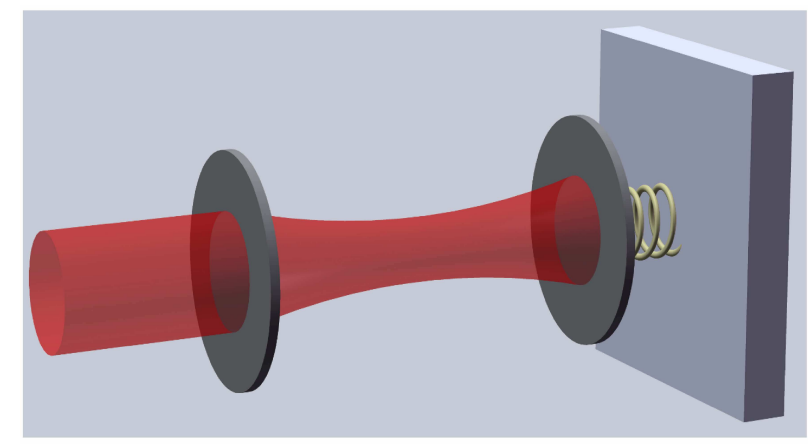

Figure 1: Schematic of a generic optomechanical system, with a laser-driven optical cavity. The left mirror is fixed and the right mirror is movable.

very weak. To overcome this problem, optical cavities are employed, which resonantly enhance the intracavity light intensity so that the optical forces become pronounced. For example, in a Fabry-Pérot (FP) cavity consisting of a fixed mirror and a movable mirror attached to a spring (Fig. 1), light is reflected multiple times between the two mirrors, and thus the cavity field builds up, resulting largely enhanced optical force exerting on the movable mirror. The study of this field named as cavity optomechanics was pioneered by Braginsky and co-workers [6, 7] with microwave cavities. Later, experiments in the optical domain demonstrated the optomechanical bistability phenomenon [8], where a macroscopic mirror had two stable equilibrium positions under the action of cavity-enhanced radiation pressure force. Further experiments observed optical feedback cooling of mechanical motion based on precise measurement and active feedback [9, 10], and along this line cooling to much lower temperatures was realized later [11, 12, 13]. On the other hand, after the observation of radiation-pressure induced self-oscillations (parametric instability) in optical microtoroidal cavities [14, 15, 16, passive cooling, which uses purely the intrinsic backaction effect of the cavity optomechanical system, attracted much attention in the past decade [17, 18, 19, 20, 21, 22, 23, 24, 25, 26, 27]. Moreover, recent theoretical and experimental efforts have demonstrated optomechanically induced transparency [28, 29, 30, 31, 32], optomechanical storage [33], normal mode splitting [34, 35, 36, 37, 38, 39], quantum-coherent coupling between optical modes and mechanical modes [40, 41] and state transfer at different optical wavelengths [42, 43, 44, 45, 46, 47, 48. Various experimental systems are proposed and investigated, including FP cavities [17, 18], whispering-gallery microcavities [15, 49, 50, 51], microring cavities [52], photonic crystal cavities [53, 54, 55, 56], membranes [57, 58, 59, 60], nanos- 
trings [61], nanorods [62, 63, 64], hybrid plasmonic structures [65], optically levitated particles [66, 67, 68, 69, 70, 71, 72], cold atoms [73, 74, 75] and superconducting circuits [76]. Recently much attention is also focused on the studies of single-photon strong optomechanical coupling [77, 78, 79, 80, 81, 82], single-photon transport [83, 84, 85, 86], nonlinear quantum optomechanics [87, 88, 89, 90], quadratic coupling [57, 91, 92, 93, 94, 95, 96, 97, 98], quantum superposition [99, 100, 101, entanglement [102, 103, 104, 105, 106, 107, 108, 109, 110, 111, 112, 113, squeezing [114, 115, 116, 117, 118, decoherence [119, optomechanical arrays [120, 121], quantum hybrid systems [122, 123, 124, 125, 126], Brillouin optomechanics [127, 128], high-precision measurements [129, 130, 131, 132, 133, 134] and so on.

The rapidly growing interest in cavity optomechanics is a result of the importance of this subject in both fundamental physics studies and applied science. On one hand, cavity optomechanics provides a unique platform for the study of fundamental quantum physics, for example, macroscopic quantum phenomena, decoherence and quantum-classical boundary. On the other hand, cavity optomechanics is promising for high-precision measurements of small forces, masses, displacements and accelerations. Furthermore, cavity optomechanics provides many useful tools for both classical and quantum information processing. For instance, optomechanical devices can serve as storages of information, interfaces between visible light and microwave. Optomechanical systems also serve as the "bridge" or "bus" in hybrid photonic, electronic and spintronic components, providing a routing for combining different systems to form hybrid quantum devices. A number of excellent reviews covering various topics has been published in the past [135, 136, 137, 138, 139, 140, 141, 142, 143, 144, 145, 146, 147, 148, 149, 150, 151, 152, 153, 154, 155, 156, 157].

As the first crucial step for preparing mechanical quantum states, cooling of mechanical resonators has been one of the central research interests in the past decade. Currently, it lacks a comprehensive review on most recent theoretical and experimental progresses of cavity optomechanical cooling, especially new cooling approaches for guiding future experiments. In this review we focus on this issue, addressing the quantum theory, recent experiments and new directions. The rest of this paper is organized as follows. In Sec. 2, we present the basic quantum theory of cavity optomechanical cooling. Starting from the system Hamiltonian, we introduce the linearization of the interaction. Then the methods for calculating the cooling rates 
and cooling limits are shown, including quantum noise approach and covariance approach. In Sec. 3, we review the up-to-date experimental progress towards cooling to the quantum ground state. Recent theoretical approaches for improving the cooling performance are discussed in Sec. 4. A summary is presented in Sec. 5.

\section{Quantum theory of cavity optomechanical cooling}

The basic idea of cavity optomechanical cooling is that the optical field introduces extra damping for the mechanical mode. Qualitatively, such optical damping is introduced because the optical force induced by the cavity field reacts with a finite delay time, corresponding to the photon lifetime of the cavity. Let us take a FP cavity optomechanical system as an example (Fig. 1). On one hand, when the movable mirror is at different position, the cavity field and thus the optical force. exerting on the mirror are also different. On the other hand, when the position of the movable mirror changes, the subsequent change of the cavity field requires some time-lag due to the finite photon lifetime. Therefore, the optical force also depends on the velocity of the movable mirror. This velocity-dependent optical force is similar to the friction caused by the intrinsic mechanical damping, and leads to extra damping (or amplification) of the mechanical motion. In the classical picture, for an optical damping rate $\Gamma_{\text {opt }}$, the resulting effective temperature of the mechanical mode being cooled is $T_{\text {eff }}=\gamma T /\left(\gamma+\Gamma_{\text {opt }}\right)$, where $\gamma$ is the intrinsic mechanical damping rate and $T$ is the environmental temperature. Note that the mechanical mode is selectively cooled, i. e., only the mode of interest is cooled while the bulk temperature of the mechanical object keeps unchanged. For the FP cavity case, the mechanical mode of interest is the center-of-mass motion of the movable mirror.

The above classical description of cavity optomechanical cooling is not accurate in some cases. For example, it does not predict cooling limits [158, 159, 160]. To accurately model the cooling process, in the following we provide full quantum theory of cavity optomechanical cooling. Here both the cavity field and the mechanical oscillation are described as quantized bosonic fields. Starting from the system Hamiltonian and taking the dissipations into consideration, we can write down the quantum Langevin equations and master equation to describe the system dynamics. For different parameter regimes, cooling performance can be obtained 
using different approaches.

\subsection{System Hamiltonian and linearization}

Let us consider a generic cavity optomechanical system with a single optical cavity mode coupled to a mechanical mode, which is canonically modeled as a FP cavity with one fixed mirror and one movable mirror mounted on a spring (Fig. 1). The system Hamiltonian is given by

$$
H=H_{\text {free }}+H_{\text {int }}+H_{\text {drive }}
$$

The first term $\left(H_{\text {free}}\right)$ is the free Hamiltonian of the optical and mechanical modes, described by

$$
H_{\text {free }}=\omega_{\mathrm{c}} a^{\dagger} a+\omega_{\mathrm{m}} b^{\dagger} b
$$

Here both of the optical and the mechanical modes are represented by quantum harmonic oscillators, where $a\left(a^{\dagger}\right)$ is the bosonic annihilation (creation) operator of the optical cavity mode, $b\left(b^{\dagger}\right)$ is the bosonic annihilation (creation) operator of the mechanical mode, and $\omega_{\mathrm{c}}$ $\left(\omega_{\mathrm{m}}\right)$ is the corresponding angular resonance frequency. The commutation relations satisfy $\left[a, a^{\dagger}\right]=1$ and $\left[b, b^{\dagger}\right]=1$. The displacement operator of the mechanical mode is given by $x=x_{\mathrm{ZPF}}\left(b^{\dagger}+b\right)$, where $x_{\mathrm{ZPF}}=\sqrt{\hbar /\left(2 m_{\mathrm{eff}} \omega_{\mathrm{m}}\right)}$ is the zero-point fluctuation, with $m_{\mathrm{eff}}$ being the effective mass of the mechanical mode.

The second term of Eq. (1) $\left(H_{\text {int }}\right)$ describes the optomechanical interaction between the optical mode and the mechanical mode, which is written as

$$
H_{\mathrm{int}}=g a^{\dagger} a\left(b^{\dagger}+b\right)
$$

where $g=\left[\partial \omega_{\mathrm{c}}(x) / \partial x\right] x_{\mathrm{ZPF}}$ represents the single-photon optomechanical coupling strength. This Hamiltonian can be obtained by simply considering that the cavity resonance frequency is modulated by the mechanical position and using Taylor expansion $\omega_{\mathrm{c}}(x)=\omega_{\mathrm{c}}+x \partial \omega_{\mathrm{c}}(x) / \partial x+$ $\mathcal{O}(x) \simeq \omega_{\mathrm{c}}+g\left(b^{\dagger}+b\right)$. A more rigorous and detailed derivation of this Hamiltonian can be found in Law's paper [161. Note that we focus on the radiation pressure force and the optical gradient force. For the photothermal force, the Hamiltonian can be found in [162].

The last term of Eq. (1) $\left(H_{\text {drive }}\right)$ describes the optical driving of the system. Assume that the system is excited through a coherent continuous-wave laser, and then the Hamiltonian is 
given by

$$
H_{\text {drive }}=\Omega^{*} e^{i \omega_{\text {in }} t} a+\Omega e^{-i \omega_{\text {in }} t} a^{\dagger} .
$$

Here $\omega_{\text {in }}$ is the input laser frequency and $\Omega=\sqrt{\kappa_{\mathrm{ex}} P /(\hbar \omega)} e^{i \phi}$ denotes the driving strength, where $P$ is the input laser power, $\phi$ is the initial phase of the input laser and $\kappa_{\text {ex }}$ is the inputcavity coupling rate.

In the frame rotating at the input laser frequency $\omega_{\text {in }}$, the system Hamiltonian is transformed to

$$
H=-\Delta a^{\dagger} a+\omega_{\mathrm{m}} b^{\dagger} b+g a^{\dagger} a\left(b^{\dagger}+b\right)+\left(\Omega^{*} a+\Omega a^{\dagger}\right),
$$

where $\Delta=\omega_{\mathrm{in}}-\omega_{\mathrm{c}}$ is the input-cavity detuning. The quantum Langevin equations are given by

$$
\begin{gathered}
\dot{a}=\left(i \Delta-\frac{\kappa}{2}\right) a-i g a\left(b+b^{\dagger}\right)-i \Omega-\sqrt{\kappa_{\mathrm{ex}}} a_{\mathrm{in}, \mathrm{ex}}-\sqrt{\kappa_{0}} a_{\mathrm{in}, 0}, \\
\dot{b}=\left(-i \omega_{\mathrm{m}}-\frac{\gamma}{2}\right) b-i g a^{\dagger} a-\sqrt{\gamma} b_{\mathrm{in}},
\end{gathered}
$$

where $\kappa_{0}$ is the intrinsic cavity dissipation rate; $\kappa=\kappa_{0}+\kappa_{\mathrm{ex}}$ is the total cavity dissipation rate; $\gamma$ is the dissipation rate of the mechanical mode; $a_{\mathrm{in}, 0}, a_{\mathrm{in}, \mathrm{ex}}$ and $b_{\mathrm{in}}$ are the noise operators associated with the intrinsic cavity dissipation, external cavity dissipation (input-cavity coupling) and mechanical dissipation. The correlations for these noise operators are given by

$$
\begin{gathered}
\left\langle a_{\mathrm{in}, 0}(t) a_{\mathrm{in}, 0}^{\dagger}\left(t^{\prime}\right)\right\rangle=\left\langle a_{\mathrm{in}, \mathrm{ex}}(t) a_{\mathrm{in}, \mathrm{ex}}^{\dagger}\left(t^{\prime}\right)\right\rangle=\delta\left(t-t^{\prime}\right), \\
\left\langle a_{\mathrm{in}, 0}^{\dagger}(t) a_{\mathrm{in}, 0}\left(t^{\prime}\right)\right\rangle=\left\langle a_{\mathrm{in}, \mathrm{ex}}^{\dagger}(t) a_{\mathrm{in}, \mathrm{ex}}\left(t^{\prime}\right)\right\rangle=0, \\
\left\langle b_{\mathrm{in}}(t) b_{\mathrm{in}}^{\dagger}\left(t^{\prime}\right)\right\rangle=\left(n_{\mathrm{th}}+1\right) \delta\left(t-t^{\prime}\right), \\
\left\langle b_{\mathrm{in}}^{\dagger}(t) b_{\mathrm{in}}\left(t^{\prime}\right)\right\rangle=n_{\mathrm{th}} \delta\left(t-t^{\prime}\right) .
\end{gathered}
$$

Here $n_{\text {th }}$ is the thermal phonon number given by

$$
n_{\mathrm{th}}=\left(e^{\frac{\hbar \omega_{\mathrm{m}}}{k_{\mathrm{B}} T}}-1\right)^{-1}
$$

where $T$ is the environmental temperature and $k_{\mathrm{B}}$ is Boltzmann constant. Note that for microwaves the thermal occupations should also be included in Eq. (8) and (9). Here we focus on optical frequencies and thus the thermal photon number is negligible.

Coherent laser input results in the displacements of both the optical and mechanical harmonic oscillators. For convenience, a displacement transformation is applied, i. e., $a \rightarrow a_{1}+\alpha$, 


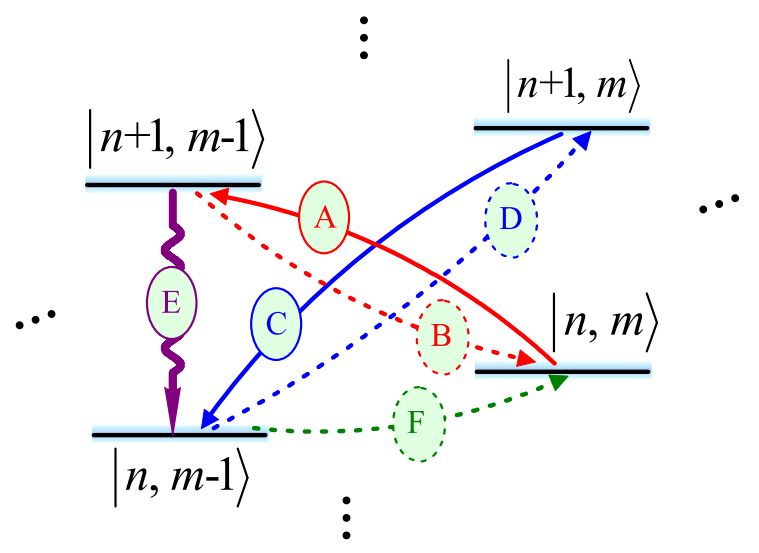

Figure 2: Level diagram of the linearized Hamiltonian (17). $|n, m\rangle$ denotes the state of $n$ photons and $m$ phonons in the displaced frame. The solid (dashed) curves with arrows correspond to the cooling (heating) processes. See text for details. Figures reproduced with permission from Ref. [163] (c) 2013 American Physical Society (APS).

$b \rightarrow b_{1}+\beta$, where $\alpha$ and $\beta$ are $c$-numbers, denoting the displacements of the optical and mechanical modes; $a_{1}$ and $b_{1}$ are the displaced operators, representing the quantum fluctuations of the optical and mechanical modes around their classical values. By separating the classical and quantum components, the quantum Langevin equations are rewritten as

$$
\begin{gathered}
\dot{\alpha}=\left(i \Delta^{\prime}-\frac{\kappa}{2}\right) \alpha-i \Omega, \\
\dot{\beta}=\left(-i \omega_{\mathrm{m}}-\frac{\gamma}{2}\right) \beta-i g|\alpha|^{2}, \\
\dot{a}_{1}=\left(i \Delta^{\prime}-\frac{\kappa}{2}\right) a_{1}-i g \alpha\left(b_{1}+b_{1}^{\dagger}\right)-i g a_{1}\left(b_{1}+b_{1}^{\dagger}\right)-\sqrt{\kappa_{\mathrm{ex}}} a_{\mathrm{in}, \mathrm{ex}}-\sqrt{\kappa_{0}} a_{\mathrm{in}, 0}, \\
\dot{b}_{1}=\left(-i \omega_{\mathrm{m}}-\frac{\gamma}{2}\right) b_{1}-i g\left(\alpha^{*} a_{1}+\alpha a_{1}^{\dagger}\right)-i g a_{1}^{\dagger} a_{1}-\sqrt{\gamma} b_{\mathrm{in}},
\end{gathered}
$$

where the optomechanical-coupling modified detuning $\Delta^{\prime}=\Delta-g\left(\beta+\beta^{*}\right)$. Under strong driving condition, the classical components dominate and the nonlinear terms $i g a_{1}\left(b_{1}+b_{1}^{\dagger}\right)$ and $i g a_{1}^{\dagger} a_{1}$ in Eqs. (15) and (16) can be neglected, respectively. Then we obtain the linearized quantum Langevin equations for $a_{1}$ and $b_{1}$, and the corresponding Hamiltonian is given by

$$
H_{L}=-\Delta^{\prime} a_{1}^{\dagger} a_{1}+\omega_{\mathrm{m}} b_{1}^{\dagger} b_{1}+\left(G a_{1}^{\dagger}+G^{*} a_{1}\right)\left(b_{1}+b_{1}^{\dagger}\right),
$$

where $G=\alpha g$ is the coherent intracavity field enhanced optomechanical coupling strength.

Initially the phonons are in a thermal equilibrium state and the thermal phonon number is $n_{\mathrm{th}}$. Then the interaction between the photons and the phonons, as described by the last 
term in Eq. (17), leads to the modification of the phonon number. Figure 2 displays the level diagram and the coupling routes among different states [163], where $|n, m\rangle$ represents the number state with $n(m)$ being the photon (phonon) number in the displaced frame. Denoted by the dashed curves, there are three kinds of heating processes: swap heating $(B)$, quantum backaction heating $(D)$ and thermal heating $(F)$. Thermal heating is an incoherent process arising from the interaction between the mechanical object and the environment. Swap heating and quantum backaction heating are the accompanying effect when radiation pressure is utilized to cool the mechanical motion, corresponding to the coherent interaction processes $a_{1} b_{1}^{\dagger}$ and $a_{1}^{\dagger} b_{1}^{\dagger}$, respectively. Swap heating emerges when the system is in the strong coupling regime which enables reversible energy exchange between photons and phonons. Meanwhile, quantum backaction heating can pose a fundamental limit for backaction cooling. The solid curves $(A$, $C$ and $E$ ) illustrate cooling processes associated with energy swapping, counter-rotating-wave interaction and cavity dissipation. In the following we derive the cooling rate and cooling limits by taking all the above processes.

\subsection{Quantum noise approach}

In the weak coupling regime, the optomechanical cooling can be analyzed using the perturbation theory, where optomechanical coupling is regarded as a perturbation to the optical field. The power spectrum of the optical force exerting on the mechanical motion $S_{F F}(\omega)$ is calculated with the absence of coupling to the mechanical resonator. Then the cooling (heating) rate is proportional to $S_{F F}\left( \pm \omega_{\mathrm{m}}\right)$, corresponding to the ability for absorbing (emitting) a phonon by the intracavity field.

From Eq. (17) we obtain the optical force acting on the mechanical motion, described by $F=-\left(G^{*} a_{1}+G a_{1}^{\dagger}\right) / x_{\mathrm{ZPF}}$. The quantum noise spectrum of the optical force is given by the Fourier transformation of the autocorrelation function $S_{F F}(\omega) \equiv \int\langle F(t) F(0)\rangle e^{i \omega t} d t$. The calculation is best performed in the frequency domain. In the absence of the optomechanical coupling, from Eq. (15) we obtain

$$
-i \omega \tilde{a}_{1}(\omega)=\left(i \Delta^{\prime}-\frac{\kappa}{2}\right) \tilde{a}_{1}(\omega)-\sqrt{\kappa_{\mathrm{ex}}} \tilde{a}_{\mathrm{in}, \mathrm{ex}}(\omega)-\sqrt{\kappa_{0}} \tilde{a}_{\mathrm{in}, 0}(\omega)
$$


which yields

$$
\tilde{a}_{1}(\omega)=\frac{\sqrt{\kappa} \tilde{a}_{\text {in }}(\omega)}{i\left(\omega+\Delta^{\prime}\right)-\frac{\kappa}{2}}
$$

where $\tilde{a}_{\text {in }}(\omega)=\sqrt{\kappa_{\mathrm{ex}} / \kappa} \tilde{a}_{\text {in,ex }}(\omega)+\sqrt{\kappa_{0} / \kappa} \tilde{a}_{\text {in }, 0}(\omega)$. Using $F(\omega)=-\left[G^{*} \tilde{a}_{1}(\omega)+G \tilde{a}_{1}^{\dagger}(\omega)\right] / x_{\mathrm{ZPF}}$, the spectral density of the optical force is obtained as

$$
S_{F F}(\omega)=\frac{\kappa|G \chi(\omega)|^{2}}{x_{\mathrm{ZPF}}^{2}}=\frac{|G|^{2}}{x_{\mathrm{ZPF}}^{2}} \frac{\kappa}{\left[\omega+\Delta^{\prime}\right]^{2}+\frac{\kappa^{2}}{4}} .
$$

The rate for absorbing and emitting a phonon by the cavity field are respectively given by

$$
A_{\mp}=S_{F F}\left( \pm \omega_{\mathrm{m}}\right) x_{\mathrm{ZPF}}^{2}=\frac{|G|^{2} \kappa}{\left[\omega_{\mathrm{m}} \pm \Delta^{\prime}\right]^{2}+\frac{\kappa^{2}}{4}} .
$$

We can also derive the spectral density of the mechanical mode $S_{b b}(\omega)$ by considering the full equations (Eq. (15) and (16)

$$
\begin{gathered}
-i \omega \tilde{a}_{1}(\omega)=\left(i \Delta^{\prime}-\frac{\kappa}{2}\right) \tilde{a}_{1}(\omega)-i G\left[\tilde{b}_{1}^{\dagger}(\omega)+\tilde{b}_{1}(\omega)\right]-\sqrt{\kappa_{\mathrm{ex}}} \tilde{a}_{\mathrm{in}, \mathrm{ex}}(\omega)-\sqrt{\kappa_{0}} \tilde{a}_{\mathrm{in}, 0}(\omega), \\
-i \omega \tilde{b}_{1}(\omega)=\left(-i \omega_{\mathrm{m}}-\frac{\gamma}{2}\right) \tilde{b}_{1}(\omega)-i\left[G^{*} \tilde{a}_{1}(\omega)+G \tilde{a}_{1}^{\dagger}(\omega)\right]-\sqrt{\gamma} \tilde{b}_{\mathrm{in}}(\omega),
\end{gathered}
$$

from which we obtain

$$
\tilde{b}_{1}(\omega) \simeq \frac{\sqrt{\gamma} \tilde{b}_{\text {in }}(\omega)-i \sqrt{\kappa}\left\{G^{*} \chi(\omega) \tilde{a}_{\text {in }}(\omega)+G \chi^{*}(-\omega) \tilde{a}_{\text {in }}^{\dagger}(\omega)\right\}}{i \omega-i\left(\omega_{\mathrm{m}}+\Sigma(\omega)\right)-\frac{\gamma}{2}} .
$$

where

$$
\begin{gathered}
\Sigma(\omega)=-i|G|^{2}\left[\chi(\omega)-\chi^{*}(-\omega)\right] \\
\chi(\omega)=\frac{1}{-i\left(\omega+\Delta^{\prime}\right)+\frac{\kappa}{2}} .
\end{gathered}
$$

In the second step of the derivation we have neglected the terms containing $\tilde{b}_{1}^{\dagger}(\omega)$, which is negligible near $\omega=\omega_{m}$. Here $\Sigma(\omega)$ represents the optomechanical self energy and $\chi(\omega)$ is the response function of the cavity mode. It shows that the optomechanical coupling leads to the modification of both the mechanical resonance frequency and the mechanical damping rate, which are termed as optical spring effect and optical damping effect, respectively. The frequency shift $\delta \omega_{\mathrm{m}}$ and the extra damping $\Gamma_{\mathrm{opt}}$ are given by

$$
\begin{gathered}
\delta \omega_{\mathrm{m}}=\Re \Sigma\left(\omega_{\mathrm{m}}\right)=|G|^{2} \Im\left[\frac{1}{-i\left(\omega_{\mathrm{m}}+\Delta^{\prime}\right)+\frac{\kappa}{2}}-\frac{1}{-i\left(\omega_{\mathrm{m}}-\Delta^{\prime}\right)+\frac{\kappa}{2}}\right], \\
\Gamma_{\mathrm{opt}}=-2 \Im \Sigma\left(\omega_{\mathrm{m}}\right)=2|G|^{2} \Re\left[\frac{1}{-i\left(\omega_{\mathrm{m}}+\Delta^{\prime}\right)+\frac{\kappa}{2}}-\frac{1}{-i\left(\omega_{\mathrm{m}}-\Delta^{\prime}\right)+\frac{\kappa}{2}}\right] .
\end{gathered}
$$




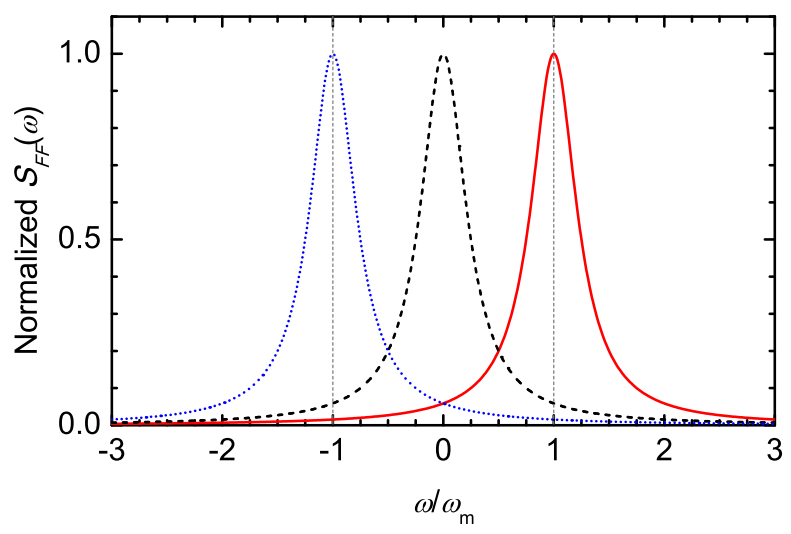

Figure 3: Normalized optical force spectrum $S_{F F}(\omega)$ for $\Delta^{\prime}=-\omega_{\mathrm{m}}$ (red solid curve), 0 (black dashed curve), and $\omega_{\mathrm{m}}$ (blue dotted curve). The dashed vertical lines denotes $\omega / \omega_{\mathrm{m}}= \pm 1$. Here $\kappa=0.5 \omega_{\mathrm{m}}$.

The spectral density of the mechanical mode is given by

$$
S_{b b}(\omega)=\int_{-\infty}^{\infty} \tilde{b}_{1}^{\dagger}(\omega) \tilde{b}_{1}\left(\omega^{\prime}\right) d \omega^{\prime}=\frac{\gamma n_{\mathrm{th}}+\kappa|G \chi(-\omega)|^{2}}{\left|i \omega-i\left(\omega_{\mathrm{m}}+\Sigma(\omega)\right)-\frac{\gamma}{2}\right|^{2}} .
$$

Figure 3 plots the normalized $S_{F F}(\omega)$ for different laser detunings. It shows that red detuning leads to $A_{-}>A_{+}$, corresponding to cooling. In this case, typically the optical damping rate $\Gamma_{\mathrm{opt}}=A_{-}-A_{+}$is much larger than the intrinsic mechanical damping rate, and then the cooling limit is obtained as

$$
n_{\mathrm{f}}=\frac{\gamma n_{\mathrm{th}}+A_{+}}{\Gamma_{\mathrm{opt}}} .
$$

Note that $n_{\mathrm{f}}^{\mathrm{c}}=\gamma n_{\mathrm{th}} / \Gamma_{\mathrm{opt}}$ is the classical cooling limit while $n_{\mathrm{f}}^{\mathrm{q}}=A_{+} / \Gamma_{\mathrm{opt}}$ corresponds to the fundamental quantum limit, as the heating rate $A_{+}$originates from the quantum backaction. This fundamental limit can be simplified as

$$
n_{\mathrm{f}}^{\mathrm{q}}=\frac{4\left(\omega_{\mathrm{m}}+\Delta^{\prime}\right)^{2}+\kappa^{2}}{-16 \omega_{\mathrm{m}} \Delta^{\prime}} .
$$

The minimal cooling limit is given by

$$
n_{\mathrm{f}, \min }^{\mathrm{q}}=\frac{1}{2}\left(\sqrt{1+\frac{\kappa^{2}}{4 \omega_{\mathrm{m}}^{2}}}-1\right),
$$

obtained when $\Delta^{\prime}=-\sqrt{\omega_{\mathrm{m}}^{2}+\kappa^{2} / 4}$. 
In particular, in the unresolved sideband regime $\left(\omega_{\mathrm{m}} \ll \kappa\right)$, the quantum limit is $n_{\mathrm{f}, \min }^{\mathrm{q}}=$ $\kappa /\left(4 \omega_{\mathrm{m}}\right)$ for $\Delta^{\prime}=-\kappa / 2$. In this case the minimum phonon number cannot reach 1 , which precludes ground state cooling. In the resolved sideband limit $\left(\omega_{\mathrm{m}} \gg \kappa\right)$, the quantum limit is simplified as $n_{\mathrm{f}, \min }^{\mathrm{q}}=\kappa^{2} /\left(16 \omega_{\mathrm{m}}^{2}\right)$ for $\Delta^{\prime}=-\omega_{\mathrm{m}}$. In this limit ground state can be achieved [158, 159].

\subsection{Covariance approach}

For the linear regime under strong driving, the mean phonon number can be computed exactly by employing the quantum master equation and solving a linear system of differential equations involving all the second-order moments. This approach holds for both weak and strong coupling regimes.

With the linearized Hamiltonian Eq. (17), the quantum master equation reads

$$
\begin{gathered}
\dot{\rho}=i\left[\rho, H_{L}\right]+\frac{\kappa}{2}\left(2 a_{1} \rho a_{1}^{\dagger}-a_{1}^{\dagger} a_{1} \rho-\rho a_{1}^{\dagger} a_{1}\right) \\
+\frac{\gamma}{2}\left(n_{\text {th }}+1\right)\left(2 b_{1} \rho b_{1}^{\dagger}-b_{1}^{\dagger} b_{1} \rho-\rho b_{1}^{\dagger} b_{1}\right)+\frac{\gamma}{2} n_{\text {th }}\left(2 b_{1}^{\dagger} \rho b_{1}-b_{1} b_{1}^{\dagger} \rho-\rho b_{1} b_{1}^{\dagger}\right)
\end{gathered}
$$

To calculate the mean phonon number, we need to determine the mean values of all the second-

order moments, $\bar{N}_{a}=\left\langle a_{1}^{\dagger} a_{1}\right\rangle, \bar{N}_{b}=\left\langle b_{1}^{\dagger} b_{1}\right\rangle,\left\langle a_{1}^{\dagger} b_{1}\right\rangle,\left\langle a_{1} b_{1}\right\rangle,\left\langle a_{1}^{2}\right\rangle$ and $\left\langle b_{1}^{2}\right\rangle$ [163, 164], which are determined by a linear system of ordinary differential equations

$$
\begin{gathered}
\frac{d}{d t} \bar{N}_{a}=-i\left(G\left\langle a_{1}^{\dagger} b_{1}\right\rangle-G^{*}\left\langle a_{1}^{\dagger} b_{1}\right\rangle^{*}+G\left\langle a_{1} b_{1}\right\rangle^{*}-G^{*}\left\langle a_{1} b_{1}\right\rangle\right)-\kappa \bar{N}_{a}, \\
\frac{d}{d t} \bar{N}_{b}=-i\left(-G\left\langle a_{1}^{\dagger} b_{1}\right\rangle+G^{*}\left\langle a_{1}^{\dagger} b_{1}\right\rangle^{*}+G\left\langle a_{1} b_{1}\right\rangle^{*}-G^{*}\left\langle a_{1} b_{1}\right\rangle\right)-\gamma \bar{N}_{b}+\gamma n_{\mathrm{th}}, \\
\frac{d}{d t}\left\langle a_{1}^{\dagger} b_{1}\right\rangle=\left[-i\left(\Delta^{\prime}+\omega_{\mathrm{m}}\right)-\frac{\kappa+\gamma}{2}\right]\left\langle a_{1}^{\dagger} b_{1}\right\rangle-i\left(G^{*} \bar{N}_{a}-G^{*} \bar{N}_{b}+G\left\langle a_{1}^{2}\right\rangle^{*}-G^{*}\left\langle b_{1}^{2}\right\rangle\right), \\
\frac{d}{d t}\left\langle a_{1} b_{1}\right\rangle=\left[i\left(\Delta^{\prime}-\omega_{\mathrm{m}}\right)-\frac{\kappa+\gamma}{2}\right]\left\langle a_{1} b_{1}\right\rangle-i\left(G \bar{N}_{a}+G \bar{N}_{b}+G+G^{*}\left\langle a_{1}^{2}\right\rangle+G\left\langle b_{1}^{2}\right\rangle\right), \\
\frac{d}{d t}\left\langle a_{1}^{2}\right\rangle=\left(2 i \Delta^{\prime}-\kappa\right)\left\langle a_{1}^{2}\right\rangle-2 i G\left(\left\langle a_{1} b_{1}\right\rangle+\left\langle a_{1}^{\dagger} b_{1}\right\rangle^{*}\right), \\
\frac{d}{d t}\left\langle b_{1}^{2}\right\rangle=\left(-2 i \omega_{\mathrm{m}}-\gamma\right)\left\langle b_{1}^{2}\right\rangle-2 i\left(G^{*}\left\langle a_{1} b_{1}\right\rangle+G\left\langle a_{1}^{\dagger} b_{1}\right\rangle\right) .
\end{gathered}
$$

Note that in the above calculation, cut-off of the density matrix is not necessary and the solutions are exact.

In the stable regime, which requires $|G|^{2}<-\left(4 \Delta^{\prime 2}+\kappa^{2}\right) \omega_{\mathrm{m}} /\left(16 \Delta^{\prime}\right)$ for red detuning $\Delta^{\prime}<0$ [165], the system finally reaches the steady state, and the derivatives in the above equations 
all become zero. Then the second-order moments in the steady state satisfy a set of algebraic equations. Under the condition $\Delta^{\prime}=-\omega_{\mathrm{m}}$ and cooperativity $C \equiv 4|G|^{2} /(\gamma \kappa) \gg 1$, the final phonon occupancy reads 163

$$
\bar{N}_{\mathrm{std}} \simeq \frac{4|G|^{2}+\kappa^{2}}{4|G|^{2}(\kappa+\gamma)} \gamma n_{\mathrm{th}}+\frac{4 \omega_{\mathrm{m}}^{2}\left(\kappa^{2}+8|G|^{2}\right)+\kappa^{2}\left(\kappa^{2}-8|G|^{2}\right)}{16 \omega_{\mathrm{m}}^{2}\left(4 \omega_{\mathrm{m}}^{2}+\kappa^{2}-16|G|^{2}\right)}
$$

Here the first term, being proportional to the environmental thermal phonon number $n_{\mathrm{th}}$, is the classical cooling limit; the second term, which does not depend on $n_{\mathrm{th}}$, corresponds to the quantum cooling limit. This quantum limit originates from the quantum backaction, consisting of both dissipation quantum backaction related to the cavity dissipation and interaction quantum backaction associated with the optomechanical interaction. In the resolved sideband case, Eq. (40) reduces to

$$
\bar{N}_{\mathrm{std}} \simeq \frac{\gamma\left(4|G|^{2}+\kappa^{2}\right)}{4|G|^{2}(\kappa+\gamma)} n_{\mathrm{th}}+\frac{\kappa^{2}+8|G|^{2}}{16\left(\omega_{\mathrm{m}}^{2}-4|G|^{2}\right)}
$$

In the weak coupling regime, it further reduces to $\bar{N}_{\mathrm{std}}^{\mathrm{wk}} \simeq \gamma n_{\mathrm{th}} /(\Gamma+\gamma)+\kappa^{2} /\left(16 \omega_{\mathrm{m}}^{2}\right)$ with $\Gamma=4|G|^{2} / \kappa$. In the strong coupling regime, $\bar{N}_{\mathrm{std}}^{\mathrm{str}} \simeq \gamma n_{\mathrm{th}} /(\kappa+\gamma)+|G|^{2} /\left[2\left(\omega_{\mathrm{m}}^{2}-4|G|^{2}\right)\right]$. In this case the classical limit is restricted by the cavity dissipation rate $\kappa$, while the interaction quantum backaction limit suffers from high coupling rate $|G|$.

To study the cooling dynamics beyond the steady state, the differential equations need to be solved to obtain the time evolution of the mean phonon number $\bar{N}_{b}$. For weak coupling, we have $\bar{N}_{b}^{\mathrm{wk}} \simeq n_{\mathrm{th}}\left(\gamma+\Gamma e^{-\Gamma t}\right) /(\gamma+\Gamma)+\left[\kappa^{2} /\left(16 \omega_{\mathrm{m}}^{2}\right)\right]\left(1-e^{-\Gamma t}\right)$, which shows that the mean phonon number decays exponentially with the cooling rate $\Gamma$. This cooling rate is limited by the coupling strength, since in the cooling route $A \rightarrow E$ as shown in Fig. 2, the energy flow from the mechanical mode to the optical mode (process $A$ ) is slower than the cavity dissipation (process $E$ ).

In the strong coupling regime, the time evolution of the mean phonon number is described by 163

$$
\begin{gathered}
\bar{N}_{b}^{\mathrm{str}}=\bar{N}_{b, 1}^{\mathrm{str}}+\bar{N}_{b, 2}^{\mathrm{str}} \\
\bar{N}_{b, 1}^{\mathrm{str}} \simeq n_{\mathrm{th}} \frac{\gamma+\frac{1}{2} e^{-\frac{\kappa+\gamma}{2} t}\left[\kappa-\gamma+(\kappa+\gamma) \cos \left(\omega_{+}-\omega_{-}\right) t\right]}{\kappa+\gamma} \\
\bar{N}_{b, 2}^{\mathrm{str}} \simeq \frac{|G|^{2}\left[1-e^{-\frac{\kappa+\gamma}{2} t} \cos \left(\omega_{+}+\omega_{-}\right) t \cos \left(\omega_{+}-\omega_{-}\right) t\right]}{2\left(\omega_{\mathrm{m}}^{2}-4|G|^{2}\right)}
\end{gathered}
$$




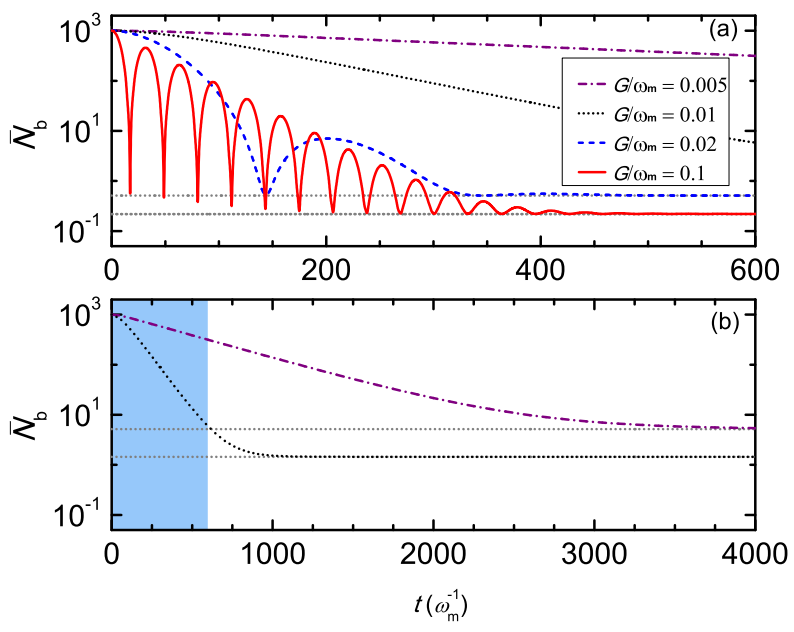

Figure 4: (a) Time evolution of mean phonon number $\bar{N}_{b}$ for $G / \omega_{\mathrm{m}}=0.005,0.01,0.02$ and 0.1 (numerical results). (b) $\bar{N}_{b}$ for $G / \omega_{\mathrm{m}}=0.005$ and 0.01 with a wider time interval. The shadowed region shows the same time interval with (a). Other parameters: $n_{\text {th }}=10^{3}, \gamma / \omega_{\mathrm{m}}=10^{-5}$, $\kappa / \omega_{\mathrm{m}}=0.05$. The dotted horizontal lines correspond to the steady-state cooling limits, given by Eq. (41). Figures reproduced with permission from Ref. [163] (c) 2013 APS.

where $\omega_{ \pm}=\sqrt{\omega_{\mathrm{m}}^{2} \pm 2|G| \omega_{\mathrm{m}}}$ are the normal eigenmode frequencies. The phonon occupancy exhibits oscillation under an exponentially-decaying envelope and can be divided into two distinguishable parts $\bar{N}_{b, 1}^{\text {str }}$ and $\bar{N}_{b, 2}^{\text {str }}$, where the first part originates from energy exchange between optical and mechanical modes, and the second part is induced by quantum backaction. $\bar{N}_{b, 1}^{\text {str }}$ reveals Rabi oscillation with frequency $\sim 2|G|$, whereas the envelopes have the same exponential decay rate $\Gamma^{\prime}=(\kappa+\gamma) / 2$ regardless of the coupling strength $|G|$. This is because, in the strong coupling regime, the cooling route $A \rightarrow E$ is subjected to the cavity dissipation (process $E$ ), which has slower rate than the energy exchange between phonons and photons (process A). This saturation prevents a higher cooling speed for stronger coupling. Figures 4(a) and (b) plot the numerical results based on the master equation for various $G$. It shows that for weak coupling the cooling rate increases rapidly as the coupling strength increases, whereas for strong coupling the envelope decay no longer increases, instead the oscillation frequency becomes larger. 


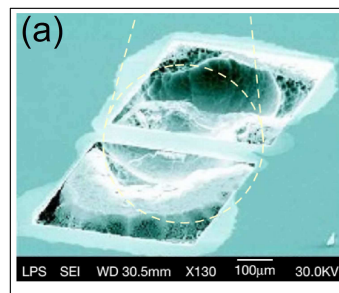

(b)

(c)
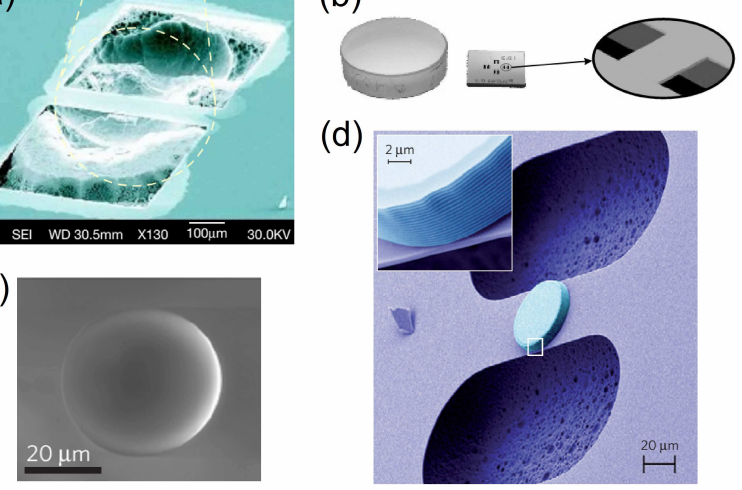

(d)

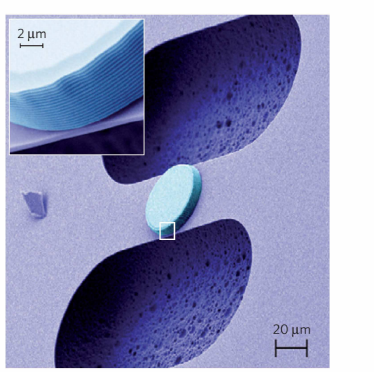

Figure 5: (a) Scanning electron microscope (SEM) image of the cantilever, a doubly clamped free-standing Bragg mirror (520 $\mu \mathrm{m}$ long, $120 \mu \mathrm{m}$ wide and $2.4 \mu \mathrm{m}$ thick) that had been fabricated by using ultraviolet excimer-laser ablation in combination with a dry-etching process. (b) Layout of the micromirror optical cavity. The microresonator mirror is etched upon a 1 cm silicon chip. The coupling mirror of the cavity is a standard low-loss silica mirror. (c) SEM image of a deformed silica microsphere. (d) SEM image of the mechanical system formed by a doubly clamped SiN beam. A circular, high-reflectivity Bragg mirror is used as the end mirror of a FP cavity. Figures reproduced with permission from: (a) Ref. [17] (c) 2006 Nature Publishing Group (NPG); (b) Ref. [18] (c) 2006 NPG; (c) Ref. [22] (c) 2009 NPG; (d) Ref. [21] (c) 2009 NPG.

\section{Recent experimental progresses}

Pioneering work of cavity optomechanical cooling dates back to 1960th by Braginsky and coworkers [6, 7], where they demonstrated the modification of mechanical damping rate as a result of the retarded nature of the cavity-enhanced optical force due to the finite cavity photon lifetime. In 2006, radiation pressure cooling was realized in three groups using different optomechanical systems, including suspended micromirrors [17, 18] (Fig. 5] (a) and (b)) and microtoroids [19]. Later in 2008, cooling in the resolved sideband regime was achieved [20]. Soon afterwards, with environmental pre-cooling under cryogenic condition, cooling to only a few phonons was demonstrated [21, 22, 23] (Fig. 5] (c) and (d)). Recently, several groups have cooled the mechanical motion close to the quantum ground state both in the microwave domain [24, 25] (Fig. 6) and in the optical domain [26, 27, 40] (Fig. 7). 

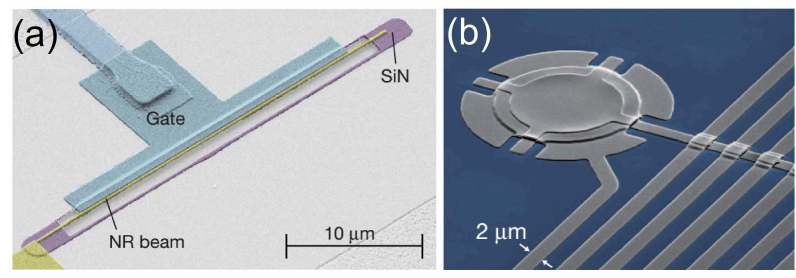

Figure 6: (a) SEM image of the Nb-Al-SiN sample. The nanomechanical resonator is $30 \mu \mathrm{m}$ long, $170 \mathrm{~nm}$ wide and $140 \mathrm{~nm}$ thick, and is formed of $60 \mathrm{~nm}$ of stoichiometric, high-stress, lowpressure chemical-vapour-deposition $\mathrm{SiN}$ and $80 \mathrm{~nm}$ of Al. (b) SEM image of the aluminium (grey) electromechanical circuit fabricated on a sapphire (blue) substrate. A 15- $\mu$ m-diameter membrane is suspended $50 \mathrm{~nm}$ above a lower electrode. Figures reproduced with permission from: (a) Ref. [24] (c) 2010 NPG; (b) Ref. 25] (c) 2011 NPG.

More specifically, in Ref. [26], mean phonon occupancy down to 0.85 quanta was achieved, with the ground state occupancy probability greater than $50 \%\left(P_{g}=0.54\right)$. In this experiment, the cavity optomechanical system consisted of a photonic crystal nanobeam resonator (Fig. 7 top panel). The carefully designed periodic patterning of the nanobeam resulted in Bragg scattering of both optical and acoustic guided waves. At the center of the nanobeam, a perturbation in the periodicity was introduced, leading to co-localized optical and mechanical resonances, which are coupled by optical gradient force. An external acoustic radiation shield consisting of a two dimensional "cross" pattern was designed to minimize the mechanical anchor damping through phononic bandgap. The optomechanical device was placed in a continuous-flow He-4 cryostat with pre-cooled environment temperature at $20 \mathrm{~K}$, corresponding to about 100 initial phonon occupancy for the mechanical mode with resonance frequency as high as $3.68 \mathrm{GHz}$. The mechanical Q-factor was $10^{5}$, corresponding to an intrinsic mechanical damping rate of $35 \mathrm{kHz}$. The optical Q-factor was $4 \times 10^{5}$, and thus the optical damping rate was $500 \mathrm{MHz}$. By fitting the measured data of mechanical damping effect, the single-photon optomechanical coupling strength is determined to be $910 \mathrm{kHz}$. With 2000 intracavity photons, the minimum mean phonon occupancy was observed to be $0.85 \pm 0.08$. The cooling was limited for higher drive powers, which resulted from the increase of the bath temperature due to optical absorption and the increase of the intrinsic mechanical damping rate induced by the generation of free carriers through optical absorption. 


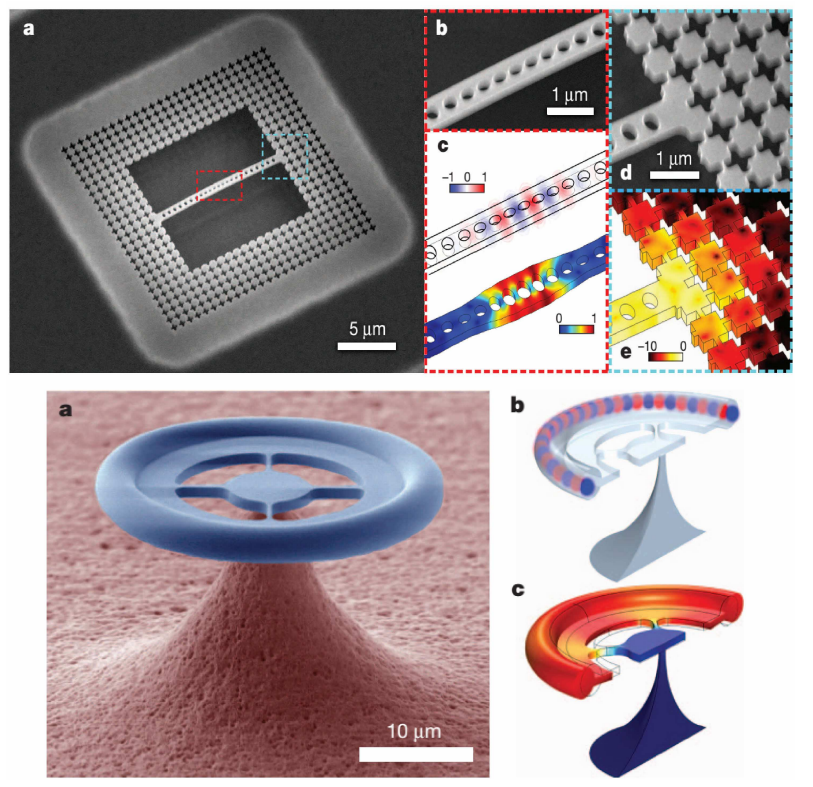

Figure 7: Top panel: Photonic nanocrystal nanobeam cavity with phononic shield. (a) Scanning electron microscope (SEM) image of the patterned silicon nanobeam and the external phononic bandgap shield. (b) Enlarged image of the central region of the nanobeam. (c) Simulation of the optical and mechanical modes. (d) Enlarged image of the nanobeam-shield interface. (e) Simulation of the localized acoustic resonance at the nanobeamCshield interface. Bottom panel: Spoke-supported microtoroidal cavity. (a) SEM image of the spoke-anchored toroidal resonator. (b) Sketch of an optical whispering gallery mode. (c) Simulation of the fundamental radial breathing mechanical mode. Figures reproduced with permission from: Top panel, Ref. [26] (c) 2011 NPG; Bottom panel, Ref. 40] (C) 2012 NPG.

In Ref. [40], a micro-optomechanical system in the form of a spoke-supported toroidal optical microcavity (Fig. 7 bottom panel) was cooled to $n_{f}=1.7$ quanta. For such microtoroidal cavity, the supported optical whispering gallery mode exhibits ultrahigh quality factor exceeding $10^{8}$, and thus the cavity decay rate reaches $\kappa / 2 \pi<10 \mathrm{MHz}$. The mechanical resonance frequency for such spoke-anchored toroidal resonator with $31 \mu \mathrm{m}$ diameter was $78 \mathrm{MHz}$. In a He-3 buffer gas cryostat with $650 \mathrm{mK}$ temperature, the mechanical resonator was pre-cooled to $\sim 200$ quanta. Through optomechanical cooling, the final mean phonon occupancy was reduced to $1.7 \pm 0.1$. Further cooling was limited by the laser reheating of the sample and the onset of normal modes. For the latter, cooling in the strong coupling regime was limited by the swap 
heating (see the next section). In this experiment, besides cooling, they also demonstrated the quantum-coherent coupling between the mechanical mode and the optical mode.

\section{New cooling approaches}

The current cooling approach as shown in Sec. 2 has achieved great successes, while there are still some major challenges. First, saturation effect appears in the strong optomechanical coupling regime as a result of swap heating. Secondly, to achieve ground state cooling, it requires the resolve sideband condition, which is stringent for many cavity optomechanical systems. In this section we review recent cooling approaches to improve the cooling performance along these directions

\subsection{Cooling in the strong coupling regime}

Recent experiments have reached the regime of strong optomechanical coupling, which is crucial for coherent quantum optomechanical manipulations. However, as mentioned in Sec. 2, strongly-coupled optomechanical cooling has predicted only limited improvement over weak coupling due to the saturation effect of the steady-state cooling rate. In Ref. [163], Liu et al. proposed to dynamically tailor the cooling and heating processes by exploiting the modulation

of cavity dissipation. In this proposal, the internal cavity dissipation is abruptly increased each time when the Rabi oscillation reaches a minimum-phonon state. At this time the system has transited from state $|n, m\rangle$ to state $|n+1, m-1\rangle$ (Fig. 2). Once a strong dissipation pulse is applied to the cavity so that the process $E$ dominates, the system will irreversibly transit from state $|n+1, m-1\rangle$ to state $|n, m-1\rangle$. The dissipation pulse has essentially behaves as a switch to halt the reversible Rabi oscillation, resulting in the suppression of the swap heating. Such dissipative cooling is verified in Figs. 8 (a) and (b), which plot the modulation scheme and the corresponding time evolution of mean phonon number $\bar{N}_{b}$. At the end of the first half Rabi oscillation cycle, $t \sim \pi /(2|G|)$, a dissipation pulse is applied. After that, the phonon number reaches and remains near the steady-state limit. Without modulation (blue dashed curve), the steady-state cooling limit is reached only after $t \simeq 400 / \omega_{\mathrm{m}}$; while with the modulation (red solid curve), it only takes $t \simeq 8 / \omega_{\mathrm{m}}$ to cool below the same limit, corresponding to 50 times 

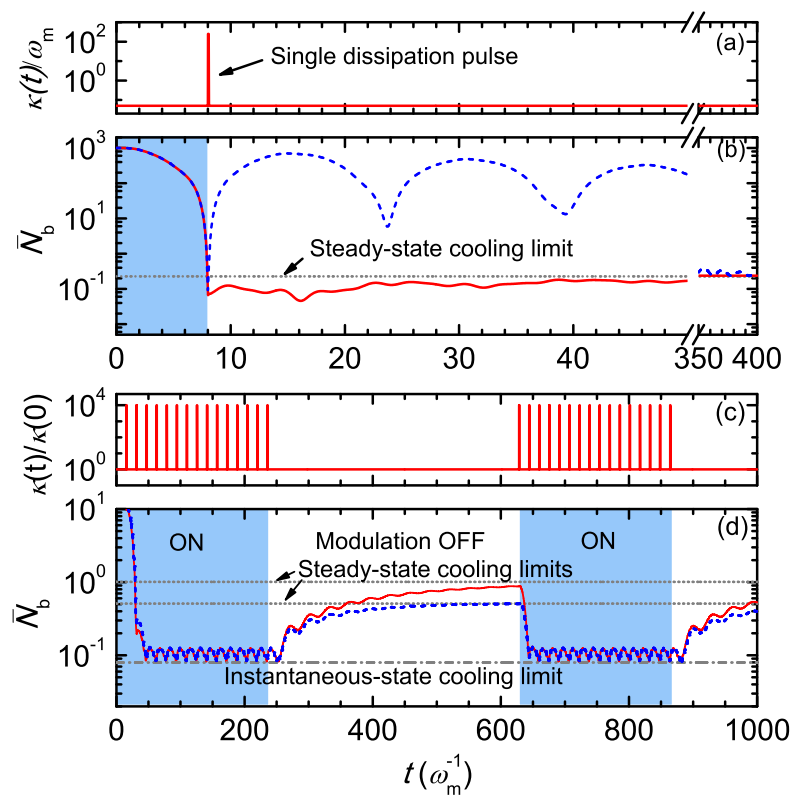

Figure 8: (a) Modulation scheme of the cavity dissipation rate $\kappa(t)$ and (b) the corresponding time evolution of mean phonon number $\bar{N}_{b}$ with (red solid curve) and without (blue dashed curve) modulation for $G / \omega_{\mathrm{m}}=0.2$ and $\kappa / \omega_{\mathrm{m}}=0.05$. (c) Modulation scheme of $\kappa(t) / \kappa(0)$ and (d) the corresponding $\bar{N}_{b}$ for $G / \omega_{\mathrm{m}}=0.1, \kappa(0) / \omega_{\mathrm{m}}=0.01$ (red solid curve) and 0.02 (blue dashed curve). In (d), the two dotted horizontal lines (from top to bottom) denoting the respective steady-state cooling limits depending on the cavity decay $\kappa(0)$; the dash-dotted line denotes the instantaneous-state cooling limit independent of $\kappa(0)$, given by Eq. (43); the "ON" and "OFF" regions corresponds that the modulation is turned on and off, respectively; the vertical coordinate range from 10 to $10^{3}$ is not shown. Other parameters: $n_{\mathrm{th}}=10^{3}$, $\gamma / \omega_{\mathrm{m}}=10^{-5}$. Figures reproduced with permission from Ref. [163] (c) 2013 APS.

faster cooling speed.

By periodically modulating the cavity dissipation so as to continuously suppress the swap heating, the phonon occupancy can be kept below the steady-state cooling limit. Each time after the dissipation pulse is applied, the photon number quickly drops to the vacuum state, which equivalently re-initializes the system. By periodic pulse application, the system will periodically re-initializes, which keeps the phonon occupancy in an instantaneous-state cooling limit as verified in Fig. 8 (c) and (d). This limit is given by [163]

$$
\bar{N}_{\mathrm{ins}} \simeq \frac{\pi \gamma n_{\mathrm{th}}}{4|G|}+\frac{\pi^{2}|G|^{4}}{\left(\omega_{\mathrm{m}}^{2}-|G|^{2}\right)\left(\omega_{\mathrm{m}}^{2}-4|G|^{2}\right)}
$$


Here the first term comes from $\bar{N}_{b, 1}^{\text {str }}$ for $t \simeq \pi /(2|G|)$, which shows a $\pi \kappa /(4|G|)$ times reduction of classical steady-state cooling limit. The second term of $\sim \pi^{2}|G|^{4} / \omega_{\mathrm{m}}^{4}$, obtained from $\bar{N}_{b, 2}^{\text {str }}$ when $t \simeq \pi / \omega_{\mathrm{m}}$, reveals that the second order term of $|G| / \omega_{\mathrm{m}}$ in quantum backaction has been removed, leaving only the higher-order terms. It is also demonstrated in Fig. 8 (c) and (d) that the modulation is switchable. If the modulation is turned on ("ON" region), the system will reach the instantaneous-state cooling limit; if the modulation is turned off ("OFF" region), the system transits back to the steady-state cooling limit.

Under frequency matching condition $\left(\omega_{+}+\omega_{-}\right) /\left(\omega_{+}-\omega_{-}\right)=k(k=3,5 \ldots)$ and $t \simeq \pi /(2|G|)$, the optimized instantaneous-state cooling limit is obtained as [163]

$$
\bar{N}_{\mathrm{ins}}^{\mathrm{opt}} \simeq \frac{\pi \kappa}{4|G|}\left[\frac{\gamma n_{\mathrm{th}}}{\kappa}+\frac{|G|^{2}}{2\left(\omega_{\mathrm{m}}^{2}-4|G|^{2}\right)}\right],
$$

which reduces both the classical and quantum steady-state cooling limits by a factor of $\pi \kappa /(4|G|)$. This reduction is significant when the system is in the deep strong coupling regime. Typically, the cooling limits can be reduced by a few orders of magnitude. For example, when $G / \omega_{\mathrm{m}}=0.3$ and $\kappa / \omega_{\mathrm{m}}=0.003$, it yields $\bar{N}_{\mathrm{std}}=3.4$, while $\bar{N}_{\mathrm{ins}}^{\mathrm{opt}}=0.03$, corresponding to more than 100 times of phonon number suppression.

\subsection{Cooling beyond the resolved sideband limit}

To loosen the stringent resolved sideband condition, a few approaches have been proposed, which can be divided into the following three directions: novel coupling mechanisms, parameter modulations and hybrid systems.

\subsubsection{Novel coupling mechanisms}

The first direction is searching for novel optomechanical coupling mechanism, for instance, dissipative coupling, where the mechanical motion couples to the cavity decay rate instead of the cavity resonance frequency [166]. In the system described in Fig. 1, the displacement of the mechanical object couples to the cavity resonance frequency $\omega(x)$, which is sometimes termed dispersive coupling. For the dissipative coupling, the displacement of the mechanical object couples to the cavity decay rate $\kappa(x)$. It was predicted [166] that this can yield novel cooling behavior, capable of reaching the quantum ground state without the resolved sideband limit. 


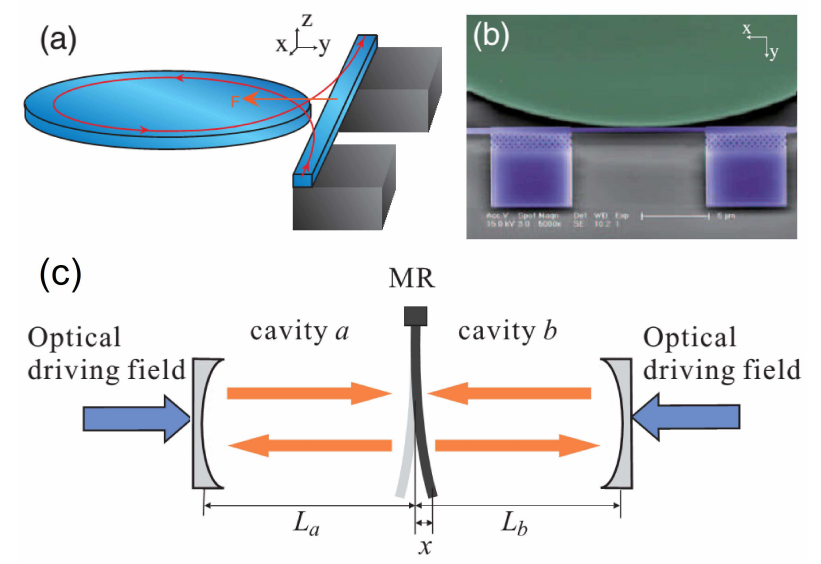

Figure 9: (a) Schematics of the microdisk-waveguide optomechanical system for dissipative coupling. (b) SEM image of the fabricated device. (c) Schematics of the three-mirror system. The movable mirror with two perfectly reflecting surfaces is placed inside a driven cavity with two transmissive fixed mirrors. Figures reproduced with permission from: (a)-(b) Ref. [168] (C) 2009 APS; (c) Ref. [176] (c) 2011 APS.

The principle is that destructive interference effect occurs between two noise sources. In the usual dispersive coupling case, the only source of backaction force noise is the number fluctuations of the cavity field, leading to Lorentzian noise spectrum. In this dissipative coupling case, the mechanical oscillator mediates the coupling between the cavity and the cavity's dissipative bath, and there are two noise sources: one is the fluctuations of the cavity field and the other is the shot noise associated with the driving laser. Note that the latter noise process is white, and thus the interference between these two noises yields a Fano line shape for the noise spectrum. Such destructive interference allows the cavity to act as an effective zero-temperature bath at a special detuning, irrespective of the resolved sideband condition. Recent analysis shows that the quantum cooling limit of cavity optomechanical system with both dispersive and dissipative couplings can be optimized [167].

Such dissipative coupling have been observed experimentally in a microdisk cavity coupled to a waveguide [168] (Fig. 9 (a) and (b)) and many theoretical analyses have been performed in various systems [169, 170, 171, 172]. 


\subsubsection{Parameter modulations}

The second direction is to introduce modulations of the system parameters, such as input laser intensity [173, 174, 175], mechanical resonance frequency [176] and other parameters [177]. In Ref. [174, 175], optimal control method were introduced, allowing ultra-efficient cooling via pulsed laser inputs. The idea is to use interference between optical pulses incident on the system, where a sequence of fast pulses adds a term to the effective optomechanical interaction Hamiltonian. In the usual continuous driving case, the interaction term has the form $x_{m} x_{c}$, while pulsed laser input generates an effective interaction term with the form $p_{m} p_{c}$, where $x_{m}$ and $p_{m}\left(x_{c}\right.$ and $\left.p_{c}\right)$ are the quadrature operators of the mechanical (optical) mode. By optimizing the pulse duration time, the total effective interaction is described by the beamsplitter Hamiltonian $x_{m} x_{c}+p_{m} p_{c} \propto a b^{\dagger}+a^{\dagger} b$. As a result, the counter-rotating-wave term is eliminated, avoiding the quantum backaction heating.

In Ref. [176], Li et al. propose a ground state cooling scheme by taking advantage of a mechanical resonator with time-dependent frequency, using a three-mirror system (Fig. 9 (c)). In this scheme, strong laser input is used to generate optical spring effect, where the effective resonance frequency of the mechanical mode is determined by the optical driving. Fast ground state cooling can be achieved by designing the trajectory of the effective frequency from the initial time to the final time.

Liao and Law have investigated the cooling performance using chirped-pulse coupling by modulating the input laser intensity and phase [178]. In this scheme, owing to the frequency modulation in chirped pulses, cooling can be realized without the need for high-precision control of the laser detuning and pulse areas.

\subsubsection{Hybrid systems}

The third direction is to construct hybrid systems, for example, couple atoms to the optical cavity. In Ref. [179], Genes et al. have described a hybrid system by coupling a two-level ensemble to the cavity optomechanical system (Fig. 10 (a) and (b)). The two-level ensemble couples to the cavity mode, creating intracavity narrow bandwidth loss or gain. It induces tailored asymmetric structure of the cavity noise spectrum interacting with the mechanical mode. As a result, This allows cooling via inhibition of the Stokes-scattering process or en- 

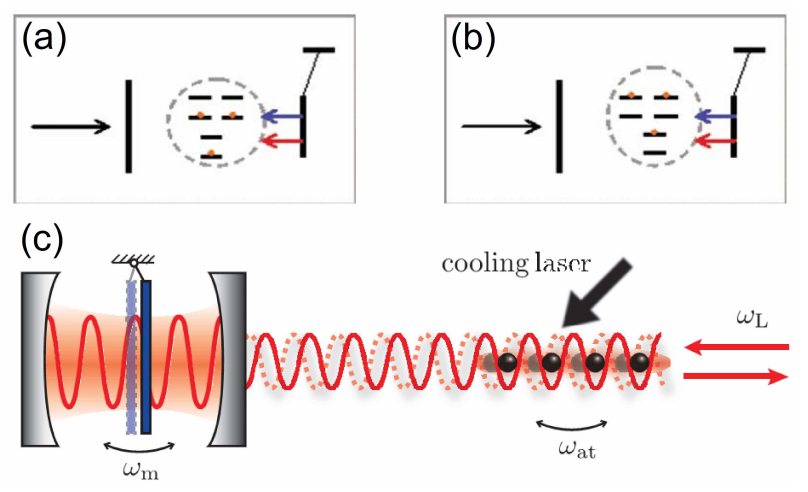

Figure 10: (a) Couple two-level atoms to the optical cavity with a movable mirror. The atoms are in the ground states. (b) Same as (a) but the atoms are in the excited states. (c) Micromechanical membrane in a cavity coupled to a distant atomic ensemble. Figures reproduced with permission from: (a)-(b) Ref. [179] (c) 2009 APS; (c) Ref. [180] (c) 2013 APS.

hancement of anti-Stokes scattering even for low-finesse optical cavities. Ground state cooling can be realized without the requirement of resolved sideband condition, as long as the loss or gain bandwidth of the two-level ensemble is narrow enough.

Similar hybrid system containing atoms are investigated in Ref. [180] (Fig. [10 (c)). Here the atomic ensemble is trapped in an optical lattice, and the center-of-mass motion instead of internal states are considered. The cavity mode mediates the interaction between the mechanical motion of the membrane and the center-of-mass motion of the atomic ensemble, leading to the effective interaction between these two mechanical modes. By laser cooling of the atomic ensemble, the membrane motion can be cooled via the effective interaction. This does not require resolved sideband conditions for the cavity, since the cavity mode acts as an intermediary.

Before conclusion, we would like to make some remarks on the influence of laser phase noise on cavity optomechanical cooling [181, 182, 183, 184, 185, 186, 187, 188], which is a technical factor that limits the cooling process. The phase noise exists in many lasers, especially in diode lasers [187]. The phase fluctuation of the cooling laser will induce the photon number fluctuation in the cavity mode. This fluctuation is equivalent to a thermal bath coupled to the mechanical resonator, and thus it limits the cooling performance. In Ref. [182], Yin has proposed a double-mode cooling configuration to reduce the influence of the laser phase noise. A whispering-gallery mode cavity with double optical modes is used, and the mechanical mode 
is coupled to both cavity modes, with the resonance frequency equal to the frequency splitting of the two cavity modes. It is shown that the phase noise effect can be strongly suppressed when the system is in the resolved sideband regime.

\section{Summary and outlook}

In summary, we have reviewed the quantum theory, recent experiments and new directions of cavity optomechanical cooling. Particularly, we summarize the new cooling scheme along two directions: cooling in the strong coupling regime and cooling beyond the resolved sideband limit. Novel cooling schemes for efficiently suppressing the thermal noise are still being explored. These schemes add complexity to the current experimental systems, and thus efforts should be taken to demonstrate these schemes in real experiments, which would be possible in the near future. There are more appealing challenges such as cooling massive mechanical objects up to kilograms and room-temperature ground state cooling of mechanical resonators.

With currently rapid experimental and theoretical advances in cavity optomechanics, it opens up new avenues to the foundations of quantum physics and applications. Quantum manipulation of macroscopic/mesoscopic mechanical objects provides a direct method to test fundamental quantum theory in a hitherto unachieved parameter regime. For example, micro mechanical structures consist of typically $10^{14}$ atoms and weigh $10^{-11}$ kilograms, while gravitational wave detectors comprise more than $10^{20}$ atoms and weigh up to several kilogram. For applications, cavity optomechanics provides new aspects for measurement with high precision and for sensing with high sensitivity. Particularly, cavity optomechanics offers a new architecture for solid-state realization of quantum information processing. The development of cavity optomechanical cooling will enable quantum manipulation of mechanical objects and generation of non-classical mechanical states, which will provide unique resources for quantum communication and quantum computation.

\section{References}

[1] Hänsch T W and Schawlow A L 1975 Opt. Commun. 1368

[2] Wineland D J and Dehmelt H 1975 Bulletin of the American Physical Society 20637 
[3] Stenholm S 1986 Rev. Mod. Phys. 58699

[4] Ashkin A 1978 Phys. Rev. Lett. 40729

[5] Metzger C H and Karrai K 2004, Nature 4321002

[6] Braginsky V B and Manukin A B 1967 Sov. Phys. JETP 25653

[7] Braginsky V B, Manukin A B and Tikhonov M Y 1970 Sov. Phys. JETP 31829

[8] Dorsel A, McCullen J D, Meystre P, Vignes E and Walther H 1983 Phys. Rev. Lett. 511550

[9] Mancini S, Vitali D, and Tombesi P, 1998 Phys. Rev. Lett. 80688

[10] Cohadon P F, Heidmann A and Pinard M 1999 Phys. Rev. Lett. 833174

[11] Kleckner D and Bouwmeester D 2006 Nature 44475

[12] Corbitt T, Wipf C, Bodiya T, Ottaway D, Sigg D, Smith N, Whitcomb S and Mavalvala N 2007 Phys. Rev. Lett. 99160801

[13] Poggio M, Degen C L, Mamin H J and Rugar D 2007 Phys. Rev. Lett. 9917201

[14] Carmon T, Rokhsari H, Yang L, Kippenberg T J and Vahala K J 2005 Phys. Rev. Lett. 94223902

[15] Kippenberg T J, Rokhsari H, Carmon T, Scherer A and Vahala K J 2005 Phys. Rev. Lett. 95 033901

[16] Rokhsari H, Kippenberg T J, Carmon T and Vahala K J 2005 Opt. Express 135293

[17] Gigan S, Böhm H R, Paternostro M, Blaser F, Langer G, Hertzberg J B, Schwab K C, Bäuerle D, Aspelmeyer M and Zeilinger A 2006 Nature 44467

[18] Arcizet O, Cohadon P F, Briant T, Pinard M and Heidmann A 2006 Nature 44471

[19] Schliesser A, Del'Haye P, Nooshi N, Vahala K J and Kippenberg T J 2006 Phys. Rev. Lett. 97 243905

[20] Schliesser A, Rivière R, Anetsberger G, Arcizet O and Kippenberg T J 2008 Nature Phys. 4415

[21] Gröblacher S, Hertzberg J B, Vanner M R, Cole G D, Gigan S, Schwab K C and Aspelmeyer M 2009 Nature Phys. 5485

[22] Park Y S and Wang H 2009 Nature Phys. 5489

[23] Schliesser A, Arcizet O, Rivère R, Anetsberger G and Kippenberg T J 2009 Nature Phys. 5509

[24] Rocheleau T, Ndukum T, Macklin C, Hertzberg J B, Clerk A A and Schwab K C 2010 Nature 46372

[25] Teufel J D, Donner T, Li D, Harlow J W, Allman M S, Cicak K, Sirois A J, Whittaker J D, Lehnert K W and Simmonds R W 2011 Nature 475359

[26] Chan J, Mayer Alegre T P, Safavi-Naeini A H, Hill J T, Krause A, Gröblacher S, Aspelmeyer M and Painter O 2011 Nature478 89 
[27] Riviere R, Deleglise S, Weis S, Gavartin E, Arcizet O, Schliesser A and Kippenberg T 2011 Phys. Rev. A 83063835

[28] Agarwal G S and Huang S 2010 Phys. Rev. A 81 041803(R)

[29] Weis S, Rivière R, Deléglise S, Gavartin E, Arcizet O, Schliesser A, Kippenberg T J 2010 Science 3301520

[30] Safavi-Naeini A H, Alegre T P M, Chan J, Eichenfield M, Winger M, Lin Q, Hill J T, Chang D E and Painter O 2011 Nature 47269

[31] Qu K and Agarwal G S 2013 Phys. Rev. A 87 031802(R)

[32] Karuza M, Biancofiore C, Bawaj M, Molinelli C, Galassi M, Natali R, Tombesi P, Giuseppe G D and Vitali D 2013 Phys. Rev. A 88013804

[33] Fiore V, Yang Y, Kuzyk M C, Barbour R, Tian L and Wang H 2011 Phys. Rev. Lett. 107133601

[34] Dobrindt J M, Wilson-Rae I and Kippenberg T J 2008 Phys. Rev. Lett. 101263602

[35] Gröblacher S, Hammerer K, Vanner M R and Aspelmeyer M 2009 Nature 460724

[36] Huang S and Agarwal G S 2009 Phys. Rev. A 80033807

[37] Akram U, Kiesel N, Aspelmeyer M and Milburn G J 2010 New J. Phys. 12083030

[38] Teufel J D, Li D, Allman M S, Cicak K, Sirois A J, Whittaker J D and Simmonds R W 2011 Nature 471204

[39] Chen H J, Mi X W 2011 Chin. Phys. B 20124203

[40] Verhagen E, Deléglise S, Weis S, Schliesser A and Kippenberg T J 2012 Nature 48263

[41] Palomaki T A, Harlow J W, Teufel J D, Simmonds R W and Lehnert K W 2013 Nature 495210

[42] Tian L and Wang H 2010 Phys. Rev. A 82053806

[43] Wang Y D and Clerk A A 2012 Phys. Rev. Lett. 108153603

[44] Tian L 2012 Phys. Rev. Lett. 108153604

[45] Barzanjeh S, Abdi M, Milburn G J, Tombesi P and Vitali D 2012 Phys. Rev. Lett. 109130503

[46] Hill J T, Safavi-Naeini A H, Chan J and Painter O 2012 Nature Comm. 31196

[47] Dong C, Fiore V, Kuzyk M C and Wang H 2012 Science 3381609

[48] Liu Y, Davanco M, Aksyuk V and Srinivasan K 2013 Phys. Rev. Lett. 110223603

[49] Tomes M and Carmon T 2009 Phys. Rev. Lett. 102113601

[50] Ma R, Schliesser A, Del'Haye P, Dabirian A, Anetsberger G and Kippenberg T J 2007 Opt. Lett. 322200

[51] Jiang X, Lin Q, Rosenberg J, Vahala K and Painter O 2009 Opt. Express 1720911 
[52] Wiederhecker G S, Chen L, Gondarenko A and Lipson M 2009 Nature 462633

[53] Eichenfield M, Camacho R, Chan J, Vahala K J and Painter O 2009 Nature 459550

[54] Eichenfield M, Chan J, Camacho R M, Vahala K J and Painter O 2009 Nature 462, 78

[55] Li Y, Zheng J, Gao J, Shu J, Aras M S and Wong C W 2010 Opt. Express 1823844

[56] Zheng J, Li Y, Aras M S, Stein A, Shepard K L and Wong C W 2012 App. Phys. Lett. 100 211908

[57] Thompson J D, Zwickl B M, Jayich A M, Marquardt F, Girvin S M and Harris J G E 2008 Nature 45272

[58] Cheung H K and Law C K 2011 Phys. Rev. A 84023812

[59] Bui C H, Zheng J, Hoch S W, Lee L Y T, Harris J G E and Wong C W 2012 App. Phys. Lett. 100021110

[60] Li H K, Liu Y C, Yi X, Zou C L, Ren X X and Xiao Y F 2012 Phys. Rev. A 85053832

[61] Anetsberger G, Arcizet O, Unterreithmeier Q P, Rivière R, Schliesser A, Weig E M, Kotthaus J P and Kippenberg T J 2009 Nature Phys. 5909

[62] Li M, Pernice W H P, Xiong C, Baehr-Jones T, Hochberg M and Tang H X 2008 Nature 456 480

[63] Favero I, Stapfner S, Hunger D, Paulitschke P, Reichel J, Lorenz H, Weig E M and Karrai K 2009 Opt. Express 1712813

[64] Zheng J, Sun X, Li Y, Poot M, Dadgar A, Shi N, Pernice W H P, Tang H X and Wong C W 2012 Opt. Express 2026484

[65] Hu Y W, Li B B, Liu Y X, Xiao Y F and Gong Q 2013 Opt. Commun. 291380

[66] Chang D E, Regal C A, Papp S B, Wilson D J, Painter O, Kimble H J and Zoller P 2010 Proc. Natl. Acad. Sci. USA 1071005

[67] Romero-Isart O, Juan M L, Quidant R and Cirac J I 2010 New J. Phys. 12033015

[68] Li T, Kheifets S and Raizen M G 2011 Nature Phys. 7527

[69] Gieseler J, Deutsch B, Quidant R and Novotny L 2012 Phys. Rev. Lett. 109103603

[70] Pender G A T, Barker P F, Marquardt F, Millen J and Monteiro T S 2012 Phys. Rev. A 85 $021802(\mathrm{R})$

[71] Yin Z Q, Li T and Feng M 2011 Phys. Rev. A 83013816

[72] Yin Z Q, Li T, Zhang X and Duan L M 2013 arXiv: 1305.1701

[73] Brennecke F, Ritter S, Donner T and Esslinger T 2008 Science 322235 
[74] Murch K W, Moore K L, Gupta S and Stamper-Kurn D M 2008 Nature Phys. 4561

[75] Zhang K, Meystre P and Zhang W 2012 Phys. Rev. Lett. 108240405

[76] Regal C A, Teufel J D and Lehnert K W 2008 Nature Phys. 4555

[77] Rabl P 2011 Phys. Rev. Lett. 107063601

[78] Nunnenkamp A, Børkje K and Girvin S M 2011 Phys. Rev. Lett. 107063602

[79] Nunnenkamp A, Børkje K and Girvin S M 2012 Phys. Rev. A 85 051803(R)

[80] Ludwig M, Safavi-Naeini A H, Painter O and Marquardt F 2012 Phys. Rev. Lett. 109063601

[81] Stannigel K, Komar P, Habraken S J M, Bennett S D, Lukin M D, Zoller P and Rabl P 2012 Phys. Rev. Lett. 109013603

[82] Xu X W, Li Y J and Liu Y X 2013 Phys. Rev. A 87025803

[83] Liao J Q, Cheung H K and Law C K 2012 Phys. Rev. A 85025803

[84] Ren X X, Li H K, Yan M Y, Liu Y C, Xiao Y F and Gong Q 2013 Phys. Rev. A 87033807

[85] Liao J Q and Law C K 2013 Phys. Rev. A 87043809

[86] Jia W Z and Wang Z D 2013 arXiv: 1308.1339

[87] Lemonde M A, Didier N and Clerk A A 2013 Phys. Rev. Lett. 111053602

[88] Børkje K, Nunnenkamp A, Teufel J D and Girvin S M 2013 Phys. Rev. Lett. 111053603

[89] Liu Y C, Xiao Y F, Chen Y L, Yu X C and Gong Q 2013 Phys. Rev. Lett. 111083601

[90] Kronwald A, and Marquardt F 2013 Phys. Rev. Lett. 111133601

[91] Bhattacharya M, Uys H and Meystre P 2008 Phys. Rev. A 77033819

[92] Sankey J C, Yang C, Zwickl B M, Jayich A M and Harris J G E 2010 Nature Phys. 6707

[93] Clerk A A, Marquardt F and Harris J G E 2010 Phys. Rev. Lett. 104213603

[94] Nunnenkamp A, Børkje K, Harris J G E and Girvin S M 2010 Phys. Rev. A 82 021806(R)

[95] Vanner M R 2011 Phys. Rev. X 1021011

[96] Buchmann L F, Zhang L, Chiruvelli A and Meystre P 2012 Phys. Rev. Lett. 108210403

[97] Deng Z J, Li Y, Gao M and Wu C W 2012 Phys. Rev. A 85025804

[98] Liao J Q and Nori F 2013 Phys. Rev. A 88023853

[99] Romero-Isart O, Pflanzer A C, Blaser F, Kaltenbaek R, Kiesel N, Aspelmeyer M and Cirac J I 2011 Phys. Rev. Lett. 107020405

[100] Romero-Isart O 2011 Phys. Rev. Lett. 84052121

[101] Pepper B, Ghobadi R, Jeffrey E, Simon C, and Bouwmeester C 2012 Phys. Rev. Lett. 109023601 
[102] Vitali D, Gigan S, Ferreira A, Böhm H R, Tombesi P, Guerreiro A, Vedral V, Zeilinger A and Aspelmeyer M 2007 Phys. Rev. Lett. 98030405

[103] Yin Z Q and Han Y J 2009 Phys. Rev. A 79024301

[104] Zou C L, Zou X B, Sun F W, Han Z F and Guo G C 2011 Phys. Rev. A 84032317

[105] Joshi C, Larson J, Jonson M, Andersson E and Öhberg P 2012 Phys. Rev. A 85033805

[106] Akram U, Munro W, Nemoto K and Milburn G J 2012 Phys. Rev. A 86042306

[107] Tian L 2013 Phys. Rev. Lett. 110233602

[108] Wang Y D and Clerk A A 2013 Phys. Rev. Lett. 110253601

[109] Xu X W, Zhao Y J and Liu Y X 2013 Phys. Rev. A 88022325

[110] Li H K, Ren X X, Liu Y C and Xiao Y F 2013 arXiv: 1306.1035

[111] Mi X W, Bai J X, Li D J 2012 Chin. Phys. B 21030303

[112] Ma Y H, Zhou L 2013 Chin. Phys. B 22024204

[113] Zhang D, Zhang X P, Zheng Q 2013 Chin. Phys. B 22064206

[114] Jähne K, Genes C, Hammerer K, Wallquist M, Polzik E S and Zoller P 2009 Phys. Rev. A 79 063819

[115] Seok H, Buchmann L F, Singh S, Steinke S K and Meystre P 2012 Phys. Rev. A 85033822

[116] Brooks D W C, Botter T, Schreppler S, Purdy T P, Brahms N and Stamper-Kurn D M 2012 Nature 488476

[117] Safavi-Naeini A H, Gröblacher S, Hill J T, Chan J, Aspelmeyer M and Painter O 2013 Nature 500185

[118] Purdy T P, Yu P L, Peterson R W, Kampel N S and Regal C A 2013 Phys. Rev. X 3031012

[119] Blencowe M P 2013 Phys. Rev. Lett. 111021302

[120] Ludwig M and Marquardt F 2013 Phys. Rev. Lett. 111073603

[121] Schmidt M, Ludwig M and Marquardt F 2012 New J. Phys. 14125005

[122] Hammerer K, Wallquist M, Genes C, Ludwig M, Marquardt F, Treutlein P, Zoller P, Ye J and Kimble H J 2009 Phys. Rev. Lett. 103063005

[123] Wallquist M, Hammerer K, Zoller P, Genes C, Ludwig M, Marquardt F, Treutlein P, Ye J and Kimble H J 2010 Phys. Rev. A 81023816

[124] Stannigel K, Rabl P, Sørensen A S, Zoller P and Lukin M D 2010 Phys. Rev. Lett. 105220501

[125] Stannigel K, Rabl P, Sørensen A S, Lukin M D and Zoller P 2011 Phys. Rev. A 84042341

[126] Habraken S J M, Stannigel K, Lukin M D, Zoller P and Rabl P 2012 New J. Phys. 14115004 
[127] Bahl G, Tomes M, Marquardt F and Carmon T 2012 Nature Phys. 8203

[128] Bahl G, Kim K H, Lee W, Liu J, Fan X and Carmon T 2013 Nature Comm. 41994

[129] LaHaye M D, Buu O, Camarota B and Schwab K C 2004 Science 30474

[130] Teufel J D, Donner R, Castellanos-Beltran M A, Harlow J W and Lehnert K W 2009 Nature Nanotech. 4820

[131] Krause A G, Winger M, Blasius T D, Lin Q and Painter O 2012 Nature Photon. 6768

[132] Forstner S, Prams S, Knittel J, van Ooijen E D, Swaim J D, Harris G I, Szorkovszky A, Bowen W P and Rubinsztein-Dunlop H 2012 Phys. Rev. Lett. 108120801

[133] Purdy T P, Peterson R W and Regal C A 2013 Science 339801

[134] Huang P, Wang P, Zhou J, Wang Z, Ju C, Wang Z, Shen Y, Duan C and Du J 2013 Phys. Rev. Lett. 110227202

[135] Kippenberg T J and Vahala K J 2007 Opt. Express 1517172

[136] Kippenberg T J and Vahala K J 2008 Science 3211172

[137] Marquardt F, Clerk A A and Girvin S M 2008 J. Mod. Opt. 553329

[138] Genes C, Mari A, Vitali D and Tombesi P 2009 Adv. At. Mol. Opt. Phys. 5733

[139] Marquardt F and Girvin S M 2009 Physics 240

[140] Favero I and Karrai K 2009 Nature Photon. 3201

[141] van Thourhout D and Roels J 2010 Nature Photon. 4211

[142] Schliesser A and Kippenberg T J 2010 Adv. At. Mol. Opt. Phys. 58207

[143] Aspelmeyer M, Gröblacher S, Hammerer K and Kiesel N 2010 J. Opt. Soc. Am. B 27 A189

[144] Zhang K Y, Zhou L, Dong G J and Zhang W P 2011 Front. Phys. 6237

[145] Milburn G J and Woolley M J 2011 Acta Physica Slovaca 61483

[146] Muschik C A, Krauter H, Hammerer K and Polzik E S 2011 Quantum Information Processing 10839

[147] Regal C A and Lehnert K W 2011 J. Phys. Conf. Ser. 264012025

[148] Ma J and Povinelli M L 2012 Current Opinion in Solid State and Materials 1682

[149] Stamper-Kurn D M, arXiv:1204.4351 (2012).

[150] Poot M and van der Zant H S J 2012 Phys. Rep. 511273

[151] Aspelmeyer M, Meystre P and Schwab K 2012 Phys. Today 6529

[152] Meystre P 2013 Ann. Phys. 525215

[153] Chen Y 2013 J. Phys. B: At. Mol. Opt. Phys. 46104001 
[154] Li J J and Zhu K D Phys. Rep. 2013525223

[155] Aspelmeyer M, Kippenberg T J and Marquardt F 2013 arXiv:1303.0733

[156] Hu Y W, Xiao Y F, Liu Y C and Gong Q 2013 Front. Phys. 8475

[157] Yin Z Q, Geraci A A and Li T 2013 Int. J. Mod. Phys. B 271330018

[158] Wilson-Rae I, Nooshi N, Zwerger W and Kippenberg T J 2007 Phys. Rev. Lett. 99093901

[159] Marquardt F, Chen J P, Clerk A A and Girvin S M 2007 Phys. Rev. Lett. 99093902

[160] Genes C, Vitali D, Tombesi P, Gigan S and Aspelmeyer M 2008 Phys. Rev. A 77033804

[161] Law C K 1995 Phys. Rev. A 512537

[162] Abdi M and Bahrampour A R 2012 Phys. Rev. A 85063839

[163] Liu Y C, Xiao Y F, Luan X and Wong C W 2013 Phys. Rev. Lett. 110153606

[164] Wilson-Rae I, Nooshi N, Dobrindt J, Kippenberg T J and Zwerger W 2008 New. J. Phys. 10 095007

[165] DeJesus E X and Kaufman C 1987 Phys. Rev. A 355288

[166] Elste F, Girvin S M and Clerk A A 2009 Phys. Rev. Lett. 102207209

[167] Weiss T and Nunnenkamp A 2013 Phys. Rev. A 88023850

[168] Li M, Pernice W H P and Tang H X 2009 Phys. Rev. Lett. 103223901

[169] Xuereb A, Schnabel R and Hammerer K 2011 Phys. Rev. Lett. 107213604

[170] Weiss T, Bruder C and Nunnenkamp A 2013 New J. Phys. 15045017

[171] Yan M Y, Li H K, Liu Y C, Jin W L and Xiao Y F 2013 Phys. Rev. A 88023802

[172] Gu W J, Li G X and Yang Y P 2013 Phys. Rev. A 88013835

[173] Vanner M R, Pikovski I, Cole G D, Kim M S, Brukner C, Hammerer K, Milburn G J and Aspelmeyer M 2011 Proc. Natl. Acad. Sci. USA 10816182

[174] Wang X, Vinjanampathy S, Strauch F W and Jacobs K 2011 Phys. Rev. Lett. 107177204

[175] Machnes S, Cerrillo J, Aspelmeyer M, Wieczorek W, Plenio M B and Retzker A 2012 Phys. Rev. Lett. 108153601

[176] Li Y, Wu L A and Wang Z D 2011 Phys. Rev. A 83043804

[177] Farace A and Giovannetti V 2012 Phys. Rev. A 86013820

[178] Liao J Q and Law C K 2011 Phys. Rev. A 84053838

[179] Genes C, Ritsch H and Vitali D 2009 Phys. Rev. A 80 061803(R)

[180] Vogell B, Stannigel K, Zoller P, Hammerer K, Rakher M T, Korppi M, Jöckel A and Treutlein P 2013 Phys. Rev. A 87023816 
[181] Diósi L 2008 Phys. Rev. A 78 021801(R)

[182] Yin Z Q 2009 Phys. Rev. A 80033821

[183] Rabl P, Genes C, Hammerer K and Aspelmeyer M 2009 Phys. Rev. A 80063819

[184] Phelps G A, Meystre P 2011 Phys. Rev. A 83063838

[185] Abdi M, Barzanjeh Sh, Tombesi P and Vitali D 2011 Phys. Rev. A 84032325

[186] Ghobadi R, Bahrampour A R and Simon C 2011 Phys. Rev. A 84063827

[187] Kippenberg T J, Schliesser A, Gorodetsky M 2013 New J. Phys. 15015019

[188] Zhang D and Zheng Q 2013 Chin. Phys. Lett. 30024213 